\section{Upper Limb After Effects of an Upper Motor Neuron Syndrome}

\section{Nathaniel H Mayer, MD*}

Director, Motor Control Analysis Laboratory, MossRehab, and Dept PM\&R, Temple University, Pennsylvania, USA

\section{Abstract \\ Objective}

To identify and illustrate a variety of skin, musculoskeletal and movement impairment after effects that are problematic for patients with an upper motor neuron syndrome.

\section{Methods}

Observation, videography, dynamic polyelectromyography (polyEMG) and clinical analysis were the basic tools used to identify a variety of after effects.

\section{Findings}

Clinical case material is presented that identifies skin lesions, problems of access, grasp dysfunction, restraint of reaching, object transport, release and problematic postural configurations. Chemodenervation with botulinum toxin, serial casting and newer types of dynamic splinting are discussed in relation to identified after effects.

\section{Conclusion}

"Spastic hemiparesis" as a term may be a useful diagnostic classifier for clinicians but, from a patient perspective, it falls short in pointing to the after effects of UMNS including many skin, musculoskeletal and dysfunctional movement findings that are of considerable concern to patients. This paper drills down to many afore-

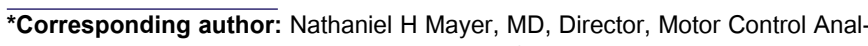
ysis Laboratory, MossRehab, Pennsylvania, USA, Tel: +1 2156636681; E-mail: nmayer@einstein.edu

Citation: Mayer NH (2018) Upper Limb After Effects of an Upper Motor Neuron Syndrome 4: 027.

Received: May 11, 2018; Accepted: August 08, 2018; Published: August 22, 2018

Copyright: (C) 2018 Mayer NH, This is an open-access article distributed under the terms of the Creative Commons Attribution License, which permits unrestricted use, distribution, and reproduction in any medium, provided the original author and source are credited. mentioned after effects in order to broaden the concept of UMNS management.

Keywords: After effects; Spastic hemiparesis; Upper motor neuron syndrome

\section{Introduction}

According to Lance's classical definition, spasticity is only one of a number of positive signs and phenomena that emerge in patients with an Upper Motor Neuron Syndrome (UMNS) [1]. However, for many years, medical treatment of patients with UMNS has focused on the management of spasticity [2]. Such a focus may have come about because the clinical finding of spasticity is relatively easy to identify and grade at the bedside. In addition, before and after measurements of spasticity treated with chemodenervation agents such as botulinum neurotoxins have demonstrated objective lessening of the clinical finding of spasticity $[3,4]$, boosting medical treatment of spasticity as the major treatment of patients with UMNS.

From a different perspective that reflects a net balance of forces generated by the interaction of 'positive' signs (involuntary muscle overactivity) and 'negative' signs (voluntary muscle underactivity) acting across joints, the after effects of UMNS such as skin problems, musculoskeletal deformity and movement impairment are of great concern to patients [5]. These after effects are experienced differently by patients from the experience of clinicians who more often focus on the neurological and neuro-physiological aspects of UMNS. This paper, observational in nature, describes and organizes many of these after effects to convey the clinical message that it may be preferable to shift the paradigm of "management of spasticity" to a more patient-centered focus.

\section{Methods}

Images were obtained with consumer grade cameras and camcorders. Most of the presented images are patients with chronic spastic hemiparesis due to stroke (duration greater than 6 months). Some patients had chronic spastic hemiparesis associated with Traumatic Brain Injury (TBI) and will be identified as such in the text or figures. Dynamic polyelectromyography (polyEMG) records were made with $\mathrm{Ag} / \mathrm{AgCl}$ surface electrodes, (Ambu Inc, Columbia, MD). EMG signals, sampled at $1 \mathrm{kHz}$, were fed through a MyoPac differential amplifier, bandwidth filtered $(20-495 \mathrm{~Hz})$, and processed by DataPac $2 \mathrm{~K} 2$ software (Run Technologies, Mission Viejo, CA). Dynamic analysis of movement was recorded with electrogoniometry using a single axis sensor (Biometrics Ltd, Ladysmith, VA).

\section{Findings}

Skin problems commonly occur in patients with UMNS who develop musculoskeletal postures resulting from a net imbalance between voluntary agonist and involuntary antagonist forces acting across joints [6]. The clenched fist is a common pattern of UMNS dysfunction and typically develops from involuntary overactivity of extrinsic finger flexors (Flexor Digitorum Sublimis (FDS) (Figure 1), Flexor Digitorum Profundus (FDP) (Figure 2), unbalanced by 
marked paresis of extensor digitorum communis [7]. Moisture with tissue maceration can accumulate underneath tightly clenched fingers (Figures 1 and 2). Often, fingernails can be seen digging into palmar skin as seen in figure 2. A clenched fist deformity (Figure 3) can conceal detritus and maceration (Figure 4) hidden in the palm. A hyperextended wrist can also lead to a clenched fist deformity due to tenodesis flexion of the fingers (Figure 5). Though flexed wrist associated with overactive wrist flexors is more commonly seen after UMNS, a hyperextended wrist is not uncommon. The resulting clenching of the fingers into the palm by tenodesis can produce maceration, erythema and skin breakdown. Treatment of wrist extensors with neurotoxin may be more important than finger flexors in such a configuration. Figure 6 reveals skin peeling after an episode of skin inflammation concealed by a tightly clenched fist, driven by a hyperextended wrist. The patient was treated with injection of botulinum neurotoxin to the wrist extensors to reduce tenodesis-driven finger clenching and allow air to circulate through the palm. Hand washing was also facilitated by the treatment because it was easier to flex the wrist passively, manipulate the hemiparetic fingers and get into the palm during washing and drying.

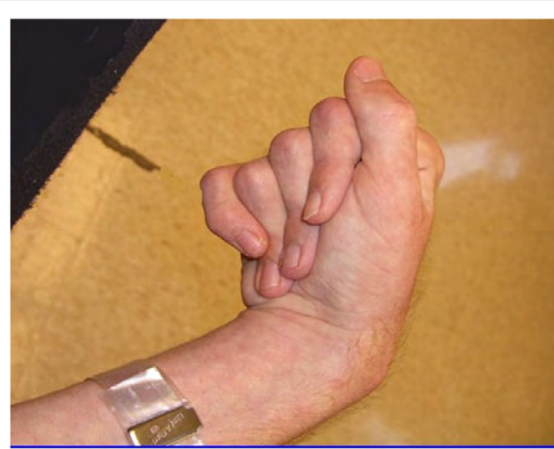

Figure 1: The Flexor Digitorum Sublimis (FDS) hand is characterized by visibility of the fingernails. The Proximal Inter-Phalangeal (PIP) joints are flexed.

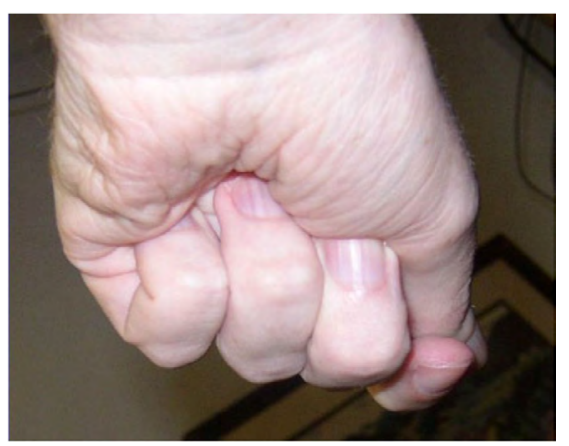

Figure 2: The Flexor Digitorum Profundus (FDP) hand is characterized by flexion of all finger joints, especially the Distal Interphalangeal (DIP) Joints.

A flexed elbow can cause skin overlap at its crease, providing a dark pocket of skin that makes for moisture accumulation and subsequent skin irritation (Figure 7). If left untreated, skin breakdown and infection can occur, especially in diabetics. Treatment consisting of chemodenervation of offending muscles with Botulinum toxin $[3,8]$ followed by use of a night resting splint or, if indicated, serial casting for a significant flexion contracture should be considered [9].

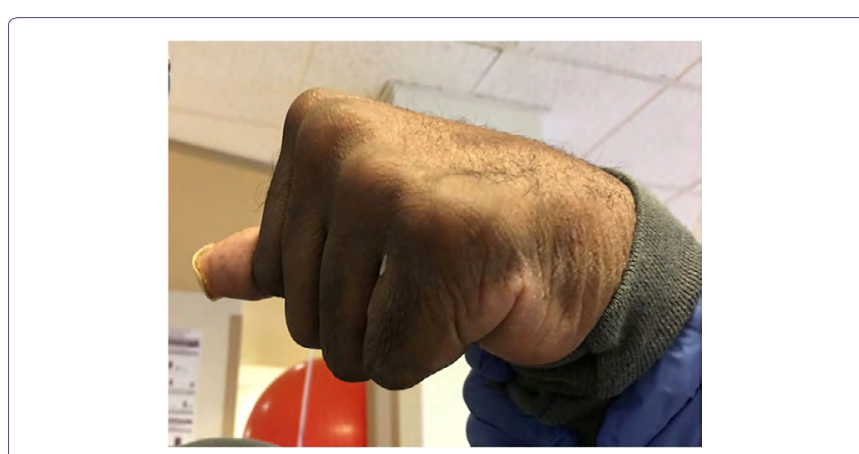

Figure 3: Clenched fist in a patient spastic hemiparesis can generate and conceal maceration and skin breakdown.

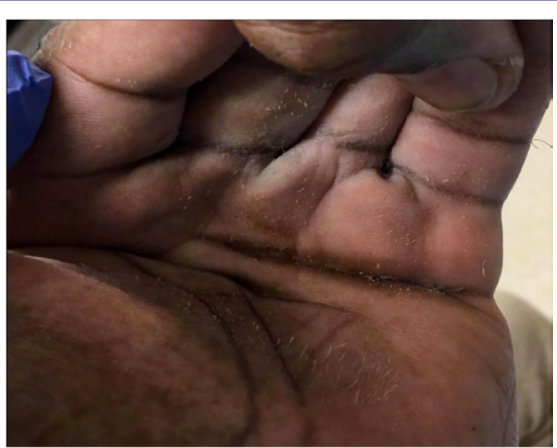

Figure 4: Detritus and maceration are present in the deep palm of a patient with clenched fist deformity.

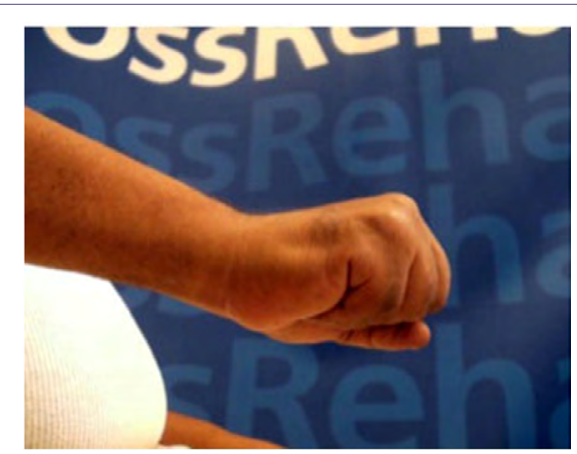

Figure 5: Hyperextended wrist leads to finger clenching by the mechanism of finger flexor tenodesis.

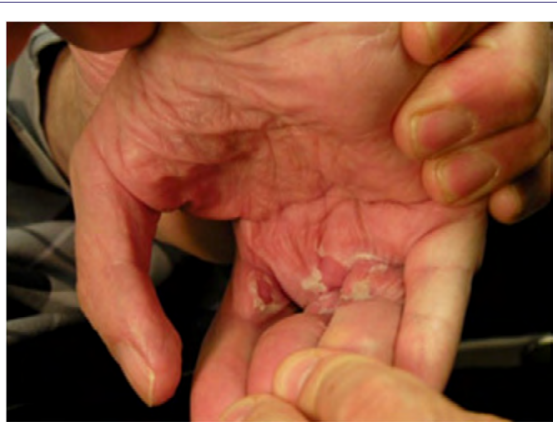

Figure 6: Skin peeling after an episode of skin inflammation concealed by a tightly clenched fist. 


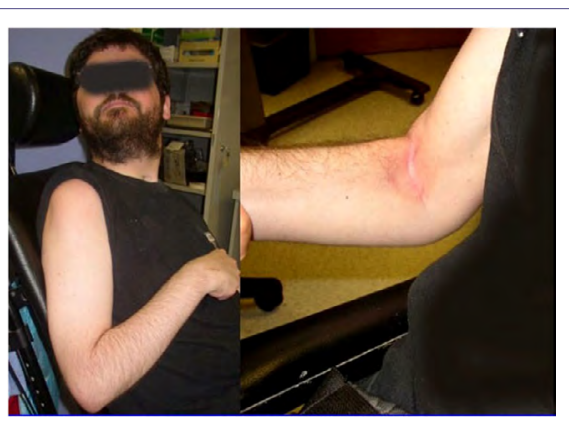

Figure 7: Patient with TBI and chronic skin overlap in the bend of the elbow resulting in skin irritation of the elbow crease.

It is important to keep in mind that more than one muscle crosses most skeletal joints so that muscle selection for chemodenervation becomes an important variable in clinical decision making [10]. Figure 8 illustrates a patient with a flexed elbow sitting "at rest". Figure 9 reveals spastic reactivity of four elbow flexors including biceps, brachialis, brachioradialis and pronator teres. The latter, though a pronator of the forearm, can also flex the elbow because of its relationship to the axis of elbow rotation. Pronator teres will flex the elbow when forearm pronation is resisted or complete (Figure 8). Figure 9 shows variability of activity in biceps, brachialis, brachioradialis and pronator teres. The elbow displacement trace shows an initial rapid phase of movement followed by a much slower prolonged phase of stretch, consistent with resistance of the elbow flexors to the examiner's stretch that has triggered spastic reflex activity in all 4 muscles. Note dystonic activity in brachialis and pronator teres after the examiner has repositioned the elbow in flexion. As an aid to muscle selection, dynamic polyEMG can help sort out the presence of involuntary muscle over activity in spastic hemiparesis [11]. Similar skin problems occur in the skin of axilla, caused by an adducted/internally rotated shoulder that maintains a clamp-like configuration of arm against trunk. Offending muscles may include pectoralis major, teres major, latissimus dorsi and subscapularis. An abducted "hanging" arm may be seen during locomotion (Figure 10). It may result in injury to arm and elbow by traumatic contact with doorways and furniture. The "hanging arm" can be injured by bumping into walls, doorways and furniture. Patients have reported to me that the "hanging" arm is particularly troublesome during shopping in a store crowded with people at holiday time.

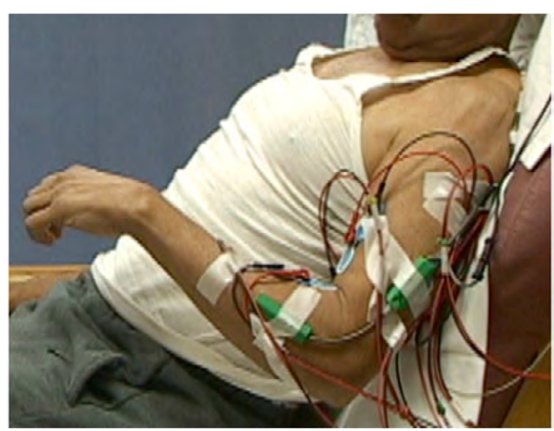

Figure 8: Flexed elbow configuration of a patient with spastic hemiparesis sitting "at rest". Instrumentation to record elbow motion and polyEMG activity is in place.

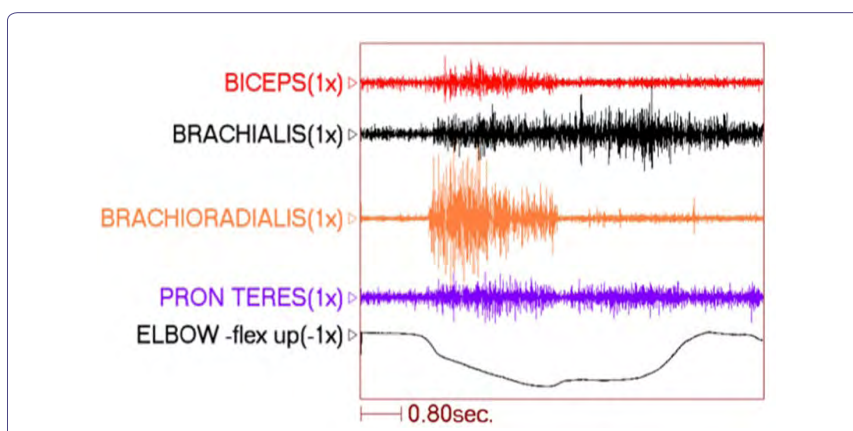

Figure 9: PolyEMG recording of passive stretch of elbow flexors in a patient with spastic hemiparesis.

Note: PRON TERES=Pronator Teres; ELBOW-flex up=Elbow flexion motion is upward for the movement trace.

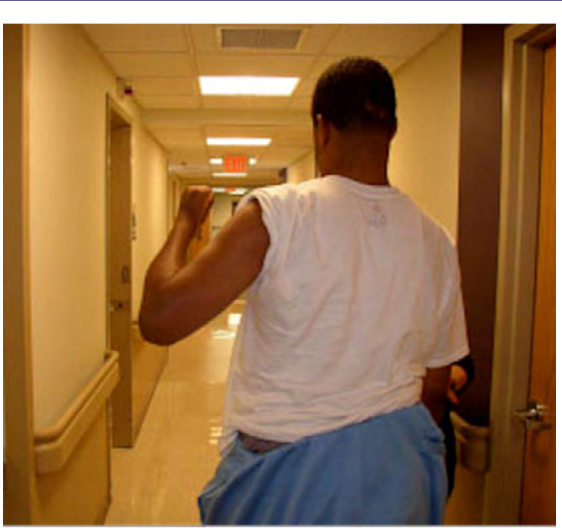

Figure 10: This patient sustained a gunshot wound of the brain resulting in a spastic hemiparesis. He exhibits an associated reaction during ambulation with shoulder abduction and flexion of the elbow.

Problems of passive daily function may be conceived as those functional acts that are ordinarily done with one upper limb that now require, in part or in whole, the assistance of the other upper limb or the assistance of a caregiver. For example, a clenched fist may need to be pried open for soaping by the unaffected limb or an aide. Figure 11 illustrates excessive flexion of the long finger DIP joint with "digging" into the palm causing a pronounced painful indentation in this patient (and sometimes frank laceration). Hand washing and especially nail care is difficult in this type of patient. Excessive pronation caused by overactive pronators aggravates nail care, even in a nail care salon. An excessively flexed or extended elbow makes for awkward and slowed sleeve dressing as does a markedly adducted/ internally rotated shoulder. In spastic hemiparesis, an affected limb with considerable hypertonia makes it difficult for the unaffected upper limb to perform manipulation of the various limb segments of the affected upper limb required by activities of daily living. Note that the ring finger in figure 11 has an FDS configuration. Mixed UMNS findings can be present in adjacent fingers of the same hand. Treatment will vary accordingly.

Most patients we see with a clenched fist retain voluntary finger flexion to a variable extent but they lose a significant degree of active finger extension. Such a 'passive' hand, i.e., a hand that doesn't open to accommodate objects of different sizes, can still be used as a holder 
of objects by using the unaffected limb and hand to insert an object into the affected hand (Figure 12). Lacking active finger extension, flexed finger configurations slow down or block access of the object into the hand (Figure 13). Neural factors and rheologic factors i.e., viscoelastic and plastic properties of muscle and connective tissue [12] play roles in entry restraint and blockage. Chemodenervation of offending superficial and deep finger flexors can improve hand as a holder function. Serial casting/splinting of finger flexor contractures is helpful as well. Hand as a holder function should be available to most persons with hemiplegia.

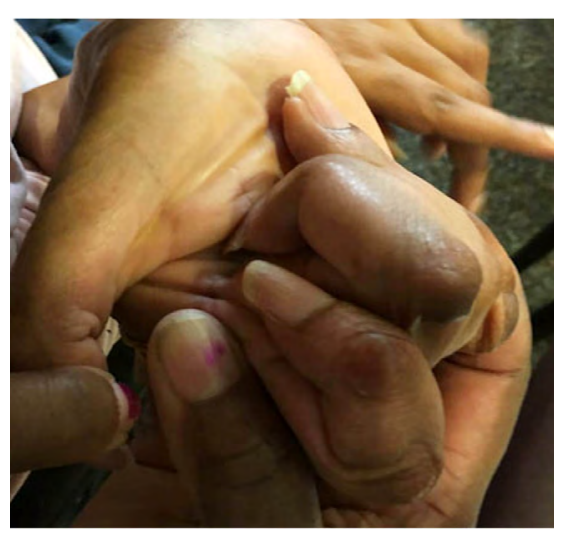

Figure 11: A case of long finger markedly digging into the skin of the palm.

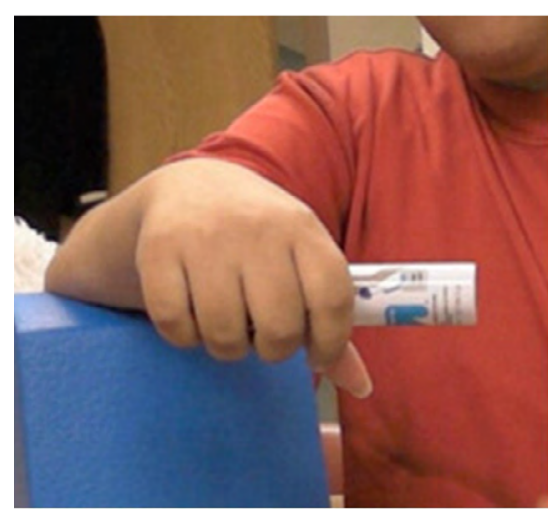

Figure 12: Illustration of 'hand as a holder'. The unaffected left hand has inserted an object into the affected hand for the purpose of holding.

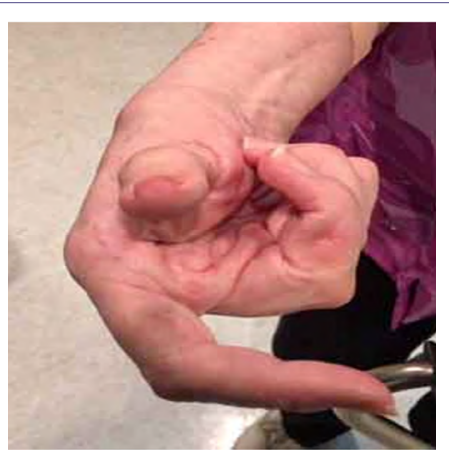

Figure 13: This hand illustrates impaired access for object entry into the hand caused by flexed long, ring and little fingers. Chemodenervation is often helpful in improving accessed by reducing finger flexor hypertonia.
Not infrequently, the index finger, thumb or long finger might curl individually or in combination during reaching and grasping. Figure 14a-d illustrate the consequences of curled index finger and thumb in a patient with spastic hemiparesis trying to pick up objects of different sizes. Focal chemodenervation of flexor digitorum profundus isolated to the index finger and thumb flexor pollicis longus should be considered. Oral anti-spastic agents are not up to the task of mitigating the forces of curling which focal blocking agents can accomplish in such cases (Figures 15 and 16).

It is not usual for a primary intrinsic plus hand to develop after an UMNS. Typically, the intrinsic plus hand may appear after neurotoxin blockor surgical lengthening of the extrinsic finger flexors. The interossei, especially the palmar interossei, are more contributory to the clinical deformity than the tinierlumbricales that are often difficult to find at the time of surgery (personal communication, Dr. Mary Ann Keenan). For the thumb, adductor pollicis, 1st dorsal interosseous and often flexor pollicis brevis combine to reduce access to the hand through the web space (Figure 17). Figure 17 shows an intrinsic plus hand characterized by flexion of the Metacarpal Phalangeal (MCP) joint, relative extension of the PIP and DIP joints as well as an adducted and flexed thumb-in-palm. Thumb Adductor Pollicis (AP), Flexor Pollicis Brevis (FPB) and first dorsal interosseous contribute to closure of the web space. The IP joint of the thumb is extended. Chemodenervation of thumb intrinsics is useful to openweb space access for object entry.

A lesion of the corticospinal tract is the most common cause of movement weakness in UMNS and current treatments largely focus on therapeutic exercise for this problem. However, spastic co-contraction of antagonists during movement often mimics weakness when antagonists exert a restraining force against agonist movement [13]. Chemo-denervation of antagonists that offend during active movement is often a useful treatment in releasing agonist muscle restraint and unmasking residual agonist strength. At the scapula, rhomboids can cause retraction during forward reaching effort that requires protraction (Figure 18).

Dynamic polyEMG is often useful in identifying antagonist muscles that contribute to restraint of movement. Figure 19 illustrates spastic co-contraction of teres major and long head of triceps during a patient's maximal effort at upward flexion of the humerus. Both of these muscles are shoulder extensors and clinically, shoulder flexor weakness is exacerbated by extensor co-contraction that acts to restrain upward flexion of the humerus. Low EMG activity is noted in latissimus dorsi, another shoulder extensor. Electromyographically, co-contraction is characterized by the onset of EMG activity in agonists and antagonists almost simultaneously before movement has begun, as seen in the figure 19 [14]. Spasticity, a phenomenon induced by stretch, typically has the onset of electromyographic activity at some point after movement has been initiated.

Figure 20 shows a patient performing alternating elbow flexion and extension movements of the elbow. His strained facial expression reveals struggle when extending his elbow.

Figure 21 shows the dynamic polyEMG of voluntary elbow flexion followed by extension performed by the patient in figure 20 . The record reveals agonist activation of triceps and anconeus during extension phase accompanied by spastic co-activation of elbow flexors during the same phase. An unsmooth movement of the elbow motion signal can be seen during extension phase with motion irregularities resulting from antagonist muscle activity. 


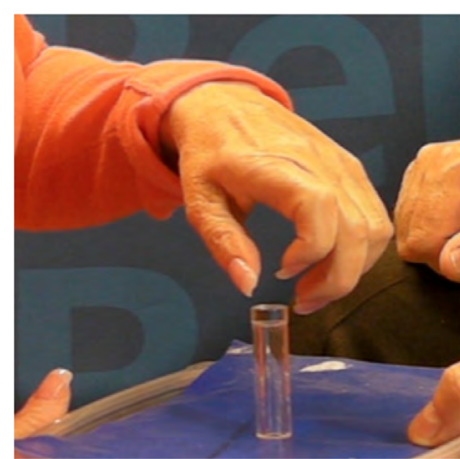

(a)

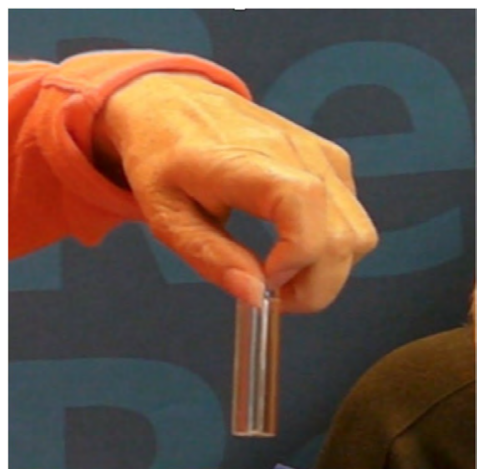

(b)

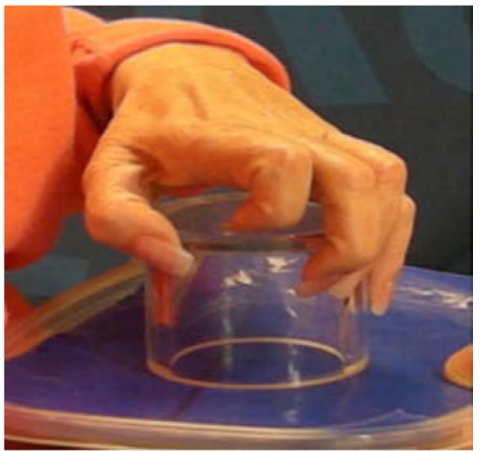

(c)

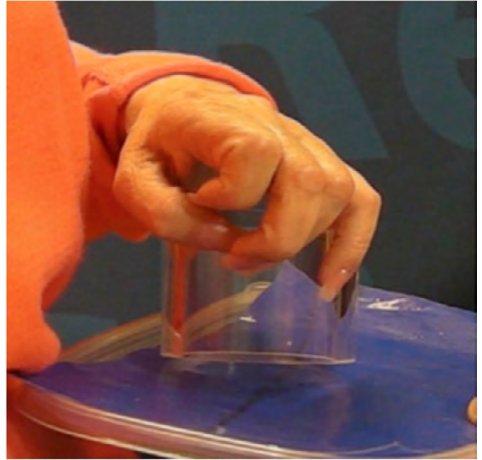

(d)

Figure 14: a) Illustrates the reach pattern with curling of the index finger prior to grasp of a 0.5 inch diameter cylinder. b) Shows the grasp pattern of this cylinder. c) Shows that a larger cylinder of 2.5 inches in diameter results in curling of the index finger on top of the cylinder, positioning it poorly for grasping. d) Shows poor grasp of a 2.5" diameter cylinder with excessive flexion of the thumb IP joint, the long finger DIP joint and the index finger which doesn't touch the cylinder.

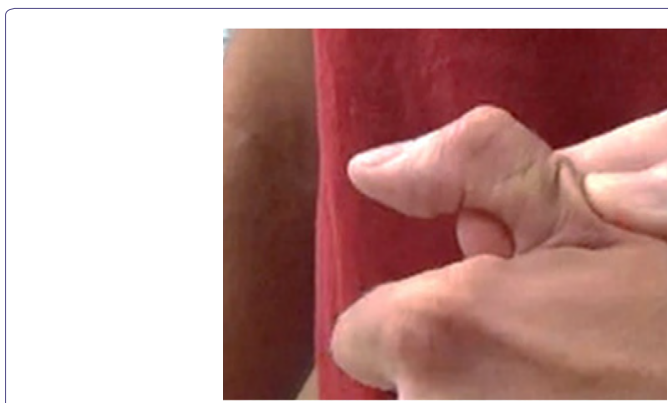

Figure 15: Flexed IP joint of the thumb that contributes to a thumb-in-palm deformity, FPL type.

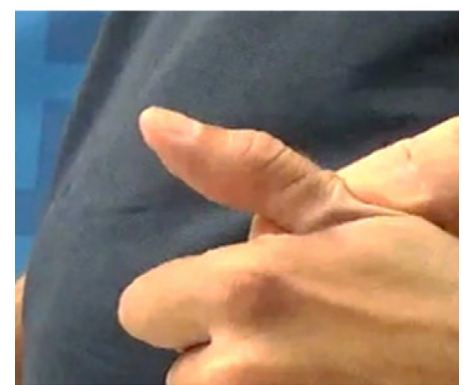

Figure 16: Active thumb extension across the IP joint after treatment of FPL with botulinum toxin.

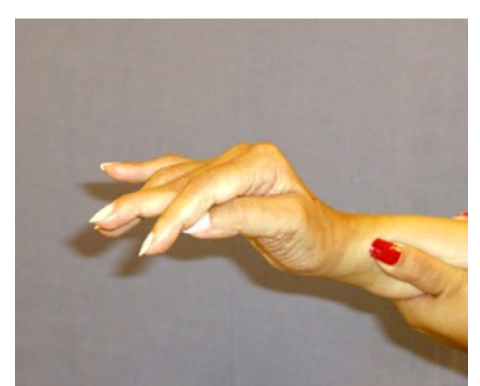

Figure 17: Hand with intrinsic plus fingers and thumb. Object entry through web space is constrained.

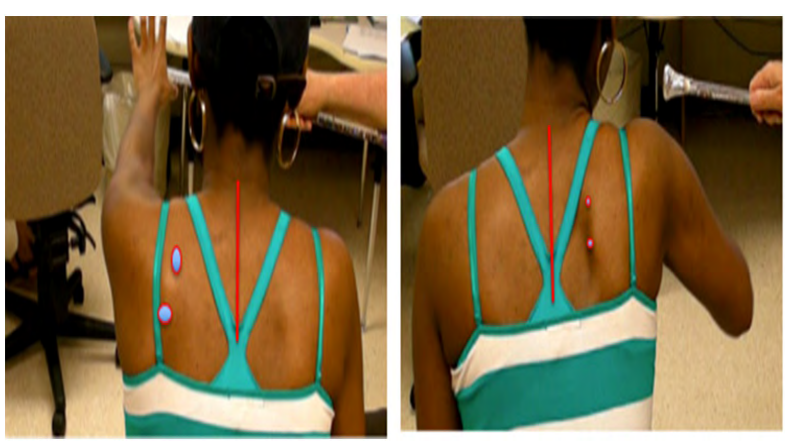

Figure 18: The image on the left reveals normal protraction of the scapula during forward reach of the left arm. The image on the right reveals scapular retraction during attempted forward reach of the right arm by this person with spastic hemiparesis. 


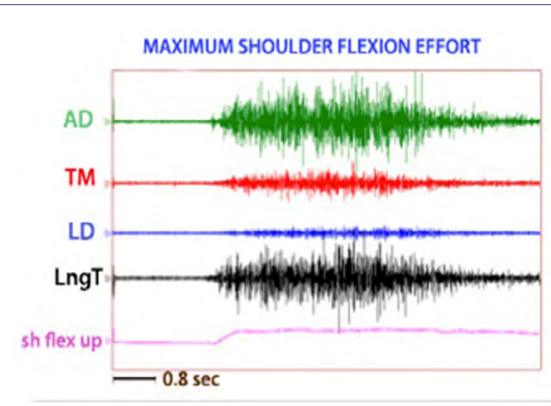

Figure 19: A patient with spastic hemiparesis was asked to make a maximum effort to flex the shoulder.

Note: $\mathrm{AD}=$ Anterior Deltoid; $\mathrm{TM}=$ Teres Major; $\mathrm{LD}=$ Latissimus Dorsi; sh flex up $=$ shoulder flexion is represented by an upward move of the electrogoniometer trace

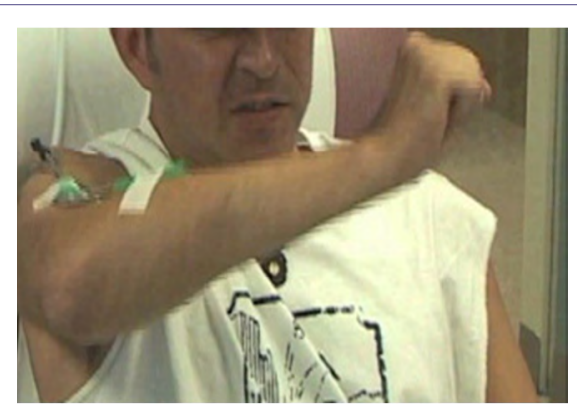

Figure 20: This patient with elbow flexor co-contraction struggles to extend his elbow. His effortful movement during active elbow extension can be seen in his strained facial expression.

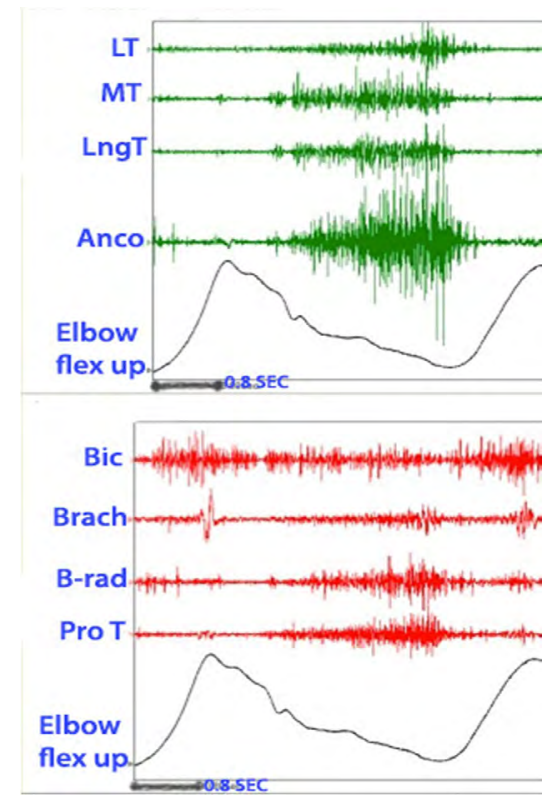

Figure 21: Dynamic polyEMG of voluntary elbow flexion followed by extension performed by the patient in figure 20 .

Note: LT=Lateral Triceps; MT=Medial Triceps; LngT=Long head Triceps; An$\mathrm{co}=$ Anconeus; Bic=Biceps; Brach=Brachialis; B-rad=Brachioradiais; Pro T=Pronator Teres; Elbow flex up=Elbow flexion is represented by an upward move of the electrogoniometer trace.
Figure 22 reveals a patient who has limited volitional supination. Figure 23 reveals the dynamic polyEMG of a voluntary supination effort made by the patient in figure 22 . There is a good recruitment pattern in the biceps as an agonist supinator. There is spastic reactivity including spikes of clonus in pronator teres and spastic reactivity in pronator quadratus. The pronators do not co-activate simultaneously with biceps but rather are delayed in their onset, suggestive of stretch reactivity. Clonus also suggests stretch reflex activity. It would appear that the supination movement triggered a spastic stretch response. The term spastic co-contraction is sometimes used to mean co-contraction with a superimposed spastic reaction triggered by agonist generated movement that stretches antagonist muscles.

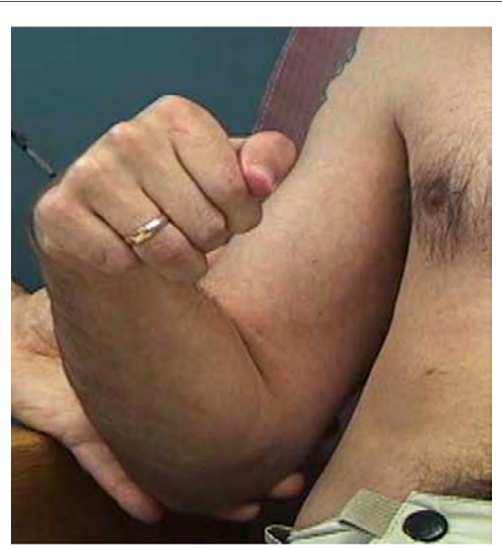

Figure 22: A patient with limited active supination. The clinical question is whether this patient has weak agonist supinators and/or spastic pronators.

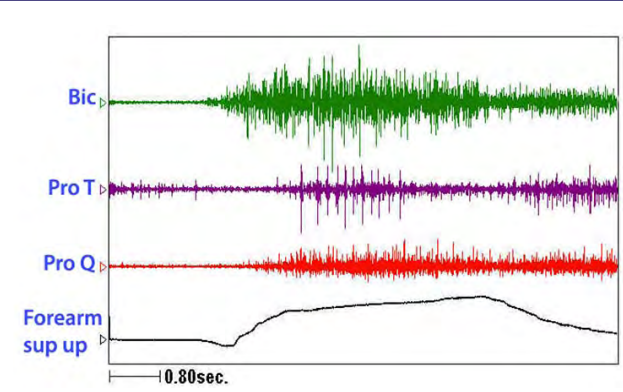

Figure 23: Dynamic polyEMG of a voluntary supination effort made by the patient in figure 22 .

Note: $\mathrm{Bic}=\mathrm{Biceps}$; Pro $\mathrm{T}=$ Pronator Teres; Pro $\mathrm{Q}=$ Pronator Quadratus (wire electrode); Forearm sup up=supination is represented by an upward move of the electrogoniometer trace.

The UMNS phenomena of spastic co-contraction and spastic reactivity are also found for wrist flexors, finger and thumb flexors, and intrinsic muscles of the hand. Once offending muscles are identified, treatment with a focal blocking agent such as botulinum toxin is clinically effective [15]. Botulinum toxin lessens the force of muscle contraction by blocking the release of acetylcholine from motor nerve terminals into the neuromuscular junction. When patients also have contracture, the neurotoxin by itself is an insufficient treatment to regain range of motion because contracture, as distinguished from contraction, is an invariant physical state of fixed tissue shortening [16]. Muscle contracture should not to be confused with muscle contraction, a dynamic, variable state of internal shortening produced by 
the sliding action of actin and myosin filaments inside a muscle fiber. Serial casting to stretch contractured muscle can be performed [17]. Surgical intervention may also be a consideration. When excessive involuntary muscle overactivity is present along with contracture, we typically opt for focal chemodenervation with botulinum toxin followed by serial casting (Figure 24). The patient in figure 24 was treated for spastic elbow flexors with botulinum toxin and serial casting was initiated about 2 weeks after injection. Botulinum neurotoxin can reduce contractile resistance to stretch imposed by the cast, making for greater tolerance of the cast by the patient and a more effective stretch of the contractured muscle. We typically wait $2+$ weeks after neurotoxin injection before the first cast is applied. Casts are changed serially once or twice a week and a typical course is 3-5 weeks of casting. We use a material known as Delta Cast Conformable which is comprised of a patented elastic fabric giving good tissue conformability.

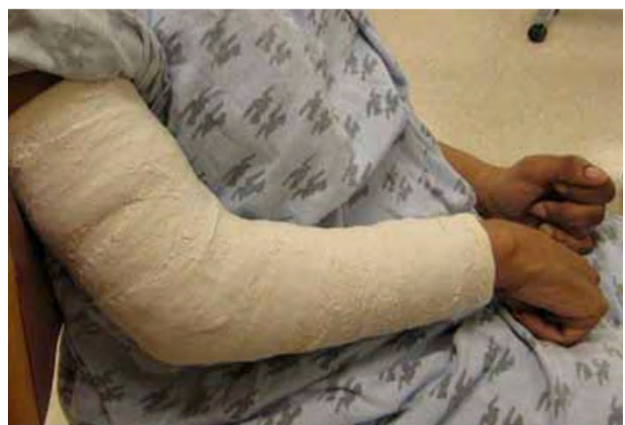

Figure 24: An example of a patient undergoing serial casting of an elbow flexion contracture.

Hand orthotics are useful to facilitate grasp and release. Figure 25 shows how a thumb-in-palm deformity affects grasp, the thumb's distal phalanx and thumb nail being the contact surface with the object. Figure 26 shows correction of the thumb-in-palm deformity with a thumbspica. Figure 27 (left image) depicts a UMNS hand with flexed fingers and thumb.

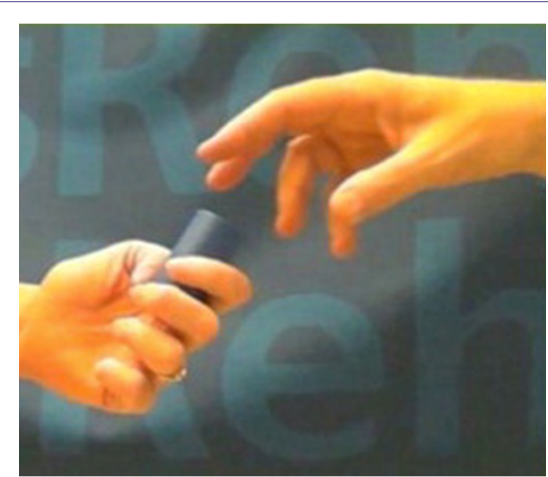

Figure 25: Image of a patient with a severe thumb-in-palm deformity of the FPL type.

The hand doesn't open because of UMNS weakness of finger and thumb extensors in addition to spastic co-contraction of extrinsic finger and thumb flexors. The right image of figure 27 shows an orthosis with a finger extension assist provided by an elastic bar [18].
The bar's spring property is loaded by finger flexion (during grasp) and then provides elastic extension for object release. In figure 27, the red arrow points to an elastic bar that applies an extension force to index and long fingers when the patient relaxes the finger flexors. A neoprene thumb sleeve has sufficient stiffness to hold the thumb out but not prevent it from flexing during grasp of an object. If finger flexors are too strong and overcome the spring's ability to extend the index and long fingers, we will treat the finger flexors with botulinum toxin to weaken them sufficiently to allow the spring to work. Finally, figure 28 illustrates the role of an aeration tunnel splint that allows air to circulate across palm skin that otherwise would be overlapped by clenched fingers trapping moisture. This splint is applied in cases where there is severe contracture that cannot be relieved by other techniques for a variety of medical or social support reasons. The size of the splint is dependent on the degree of available access into the palm.

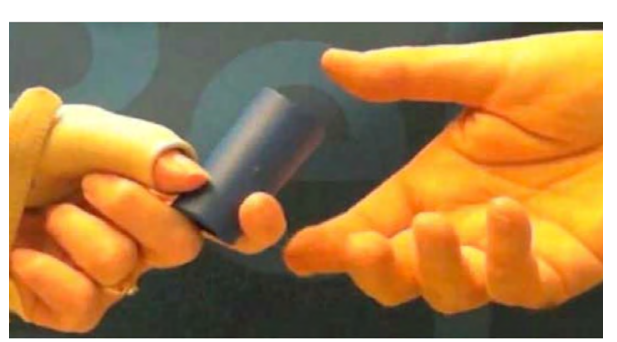

Figure 26: Application of a thumb spica orthosis that repositions the thumb with better grasp position.

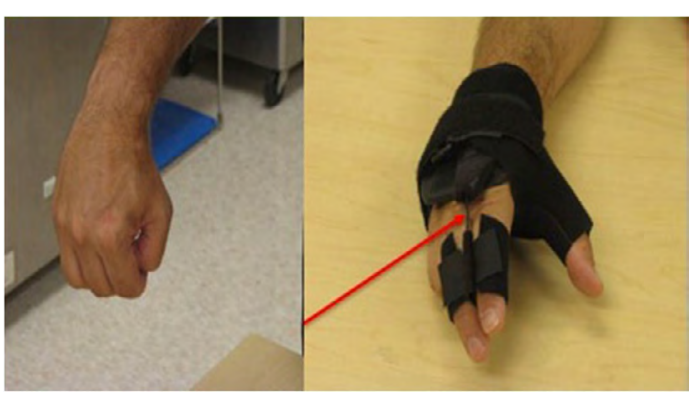

Figure 27: Patient with spastic hemiparesis who does not have active finger extension. Note difference between the closed clenched fist and the open hand facilitated by the extension assist orthosis.

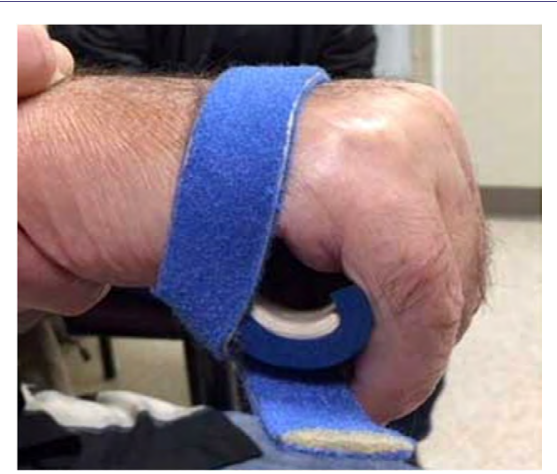

Figure 28: An aeration splint provides a tunnel for air to circulate across palm skin to keep the palm dry. 


\section{Discussion}

"Spastic hemiparesis" as a term may be a useful diagnostic classifier for clinicians but, from a patient perspective, it falls short in pointing to the many skin, musculoskeletal and dysfunctional movement after effects that are of concern to patients. UMNS phenomena in the upper limb lead to a wide variety of maladaptive skin, musculoskeletal and movement impairment after effects that affect health and function. The paper attempts to identify and illustrates problematic after effects including signs and symptoms, object entry and hand access, grasp and release dysfunction, movement and reach dysfunction. Spastic phenomena as well as paresis contribute to many after effects of UMNS but treatment emphasis, in our view, is misplaced if we say we are treating spasticity (a clinician perspective) as opposed to treating maladaptive after effects (a patient perspective). In our view, the phrase 'management of spasticity', so ubiquitous at clinical conferences, needs a semantic makeover that more clearly reflects patient concerns regarding management of skin, musculoskeletal and movement after effects. By placing a focus on many different types of UMNS after effects, we hope to have broadened the concept of UMNS management.

\section{References}

1. Lance JW (1980) Symposium synopsis. In: Feldman RG, Young RR, Koella WP (eds.). Spasticity: disordered motor control. Year Book Medical Publishers, Illinois, USA.

2. Brashear A (2016) Spasticity diagnosis and management, ( $2^{\text {nd }}$ edn). Demos Medical Publishing, New York, USA.

3. Brashear A, Gordon MF, Elovic E, Kassicieh VD, Marciniak C, et al. (2002) Intramuscular injection of botulinum toxin for the treatment of wrist and finger spasticity after a stroke. N Engl J Med 347: 395-400.

4. Simpson DM, Gracies JM, Graham HK, Miyasaki JM, Naumann M, et al. (2008) Assessment: Botulinum neurotoxin for the treatment of spasticity (an evidence-based review): Report of the therapeutics and technology assessment subcommittee of the american academy of neurology. Neurology 70: 1691-1698.

5. Mayer NH (2009) Upper limb skin and musculoskeletal consequences of the upper motor neuron syndrome. In: Jankovic J, Albanese A, Atassi M, Dolly O, Hallett M, et al. (eds.). Botulinum Toxin: Therapeutic clinical practice \& science. Saunders, Pennsylvania, USA.
6. Mayer N, Esquenazi A (2003) Muscle overactivity and movement dysfunction in the upper motoneuron syndrome. Phys Med Rehabil Clin N Am 14: 855-883.

7. Mayer NH, Esquenazi A, Childers MK (2008) Common patterns of clinical motor dysfunction. In: Brashear A, Mayer NH (eds.). Spasticity and other forms of muscle overactivity in the upper motor neuron syndrome: Etiology, evaluation, management and the role of Botulinum Toxin. WeMove, New York, USA.

8. Elia A, Graziella F, Albanese A (2009) Clinical trials of botulinuk toxin in adult spasticity. In: Jankovic J, Albanese A, Atassi M, Dolly O, Hallett M, et al. (eds.). Botulinum Toxin: Therapeutic clinical practice \& science. Saunders, Pennsylvania, USA.

9. Lannin NA, Novak I, Cusick A (2007) A systematic review of upper extremity casting for children and adults with central nervous system motor disorders. Clin Rehabil 21: 963-976.

10. Pathak MS, Nguyen HT, Graham HK, Moore AP (2006) Management of spasticity in adults: Practical application of botulinum toxin. Eur J Neurol 13: $42-50$.

11. Esquenazi A, Cioni M, Mayer NH (2008) Assessment of muscle overactivity and spasticity with dynamic polyelectromyography and motion analysis. Open Rehabil J 3: 143-148.

12. Sheean G, McGuire JR (2009) Spastic hypertonia and movement disorders: Pathophysiology, clinical presentation, and quantification. PM R 1: 827-833.

13. Gracies JM (2005) Pathophysiology of spastic paresis. II: Emergence of muscle overactivity. Muscle Nerve 31: 552-571.

14. Gracies JM (2015) Coefficients of impairment in deforming spastic paresis. Ann Phys Rehabil Med 58: 173-178.

15. Gracies JM (2004) Physiological effects of botulinum toxin in spasticity. Mov Disord 19: 120-128.

16. O’Dwyer NJ, Ada L, Neilson PD (1996) Spasticity and muscle contracture following stroke. Brain 119: 1737-1749.

17. Gharbaoui I, Katarzyna K, Cole P (2016) Spastic paralysis of the elbow and forearm. Semin Plast Surg 30: 39-44.

18. Miczak K, Padova J (2018) Muscle overactivity in the upper motor neuron syndrome: Assessment and problem solving for complex cases: The role of physical and occupational therapy. Phys Med Rehab Clinics NA 29: 529-536. 